\title{
CATS: a new station for a complete characterization of atmospheric turbulence
}

\author{
Aziz Ziad ${ }^{\mathrm{a}}$, Julien Chabéb$^{\mathrm{b}}$, Yan Fantei-Caujolle ${ }^{\mathrm{a}}$, Eric Aristidi ${ }^{\mathrm{a}}$, and Catherine Renaud ${ }^{\mathrm{a}}$ \\ a'Université Côte d'Azur, OCA, CNRS, Lagrange, Parc Valrose 06108 Nice Cedex 2, France \\ ${ }^{\mathrm{b}}$ Geoazur, IRD-UNSA-OCA-CNRS, 250 Rue A. Einstein, 06560 Valbonne, France
}

\begin{abstract}
Since autumn 2015, the Observatoire de la Côte d'Azur and the J.L. Lagrange Laboratory have equipped the Calern Observatory with a new generation station of atmospheric turbulence measurement (CATS: Calern Atmospheric Turbulence Station). The CATS station is equipped with a set of complementary instruments for monitoring atmospheric turbulence parameters. These new-generation instruments are autonomous for a fully monitoring of the turbulence within original techniques since the first meters above the ground to the borders of the atmosphere. The CATS station provides a real-time turbulence conditions over the Calern Observatory. The CATS station is a support involved in many scientific and educational projects at the Calern observatory such as an ongoing study of turbulence impact on the laser links of the MeO Laser Ranging Station, development of Adaptive Optics projects and on site training for students of the international master in Astrophysics (MAUCA) and Optics through the organization of on-sky practical works.
\end{abstract}

Keywords: Site-testing, atmospheric optics, atmospherice turbulence,turbulence monitoring.

\section{INTRODUCTION}

The Atmospheric Optics team of the he Observatoire de la Côte d'Azur and the J.L. Lagrange Laboratory, whose expertise is internationally recognized, has always played a pioneering role in the field of atmospheric turbulence characterization and site-testing in Astronomy. This expertise allowed this team to participate in the selection of the major sites of all the greatest projects of existing telescopes in particular of the 8-10 meter class: GranTeCan in the Canary Islands, the European VLT and Southern Gemini in Chile, Keck, Northern Gemini \& Subaru in Hawaii. With a unique set of instruments to probe the atmospheric turbulence, the Lagrange Laboratory was involved in the site selection of the future Extremely Large Telescopes (ELT) as the 40m European E-ELT and the $30 \mathrm{~m}$ American TMT. Our team was also in charge of the qualification of the site of Dome C in Antarctica whose potential is Astronomy is considerable. In the continuity of this long tradition, a new generation of autonomous instruments has been developed within original techniques for measuring optical turbulence since the first meters above the ground to the borders of the atmosphere. One of these instruments is the PML (Profiler of Moon Limb) measuring the vertical distribution of turbulence using lunar and solar edges. The second instrument, called G-DIMM for Generalized DIMM, is dedicated to provide wavefront parameters at ground level (seeing, outer scale, time coherence and isoplanatic angle). The third instrument is INTENSE (INdoor TurbulENce SEnsor) which is an improved version of the prototype LOTUCE (LOcal TUrbulenCe Experiment) developed by the same team for ESO. INTENSE instrument is dedicated to the characterization of optical turbulence inside a dome which can have a significant contribution to the degradation of the optical resolution of the telescope. All these instruments are part of the CATS station (Calern Atmospheric Turbulence Station) installed near the MeO telescope at Calern Observatory in the South-East of France. This station is a response to real needs in support of other projects that will give additional dynamic to the Calern Observatory. This is mainly to improve the link budget of Laser Telemetry from $\mathrm{MeO}$ station and generally free space optical links. It is also supporting projects on $\mathrm{MeO}$ and $\mathrm{C} 2 \mathrm{PU}$ telescopes to test and validate new concepts and components in order to overcome the current limitations of existing Adaptive Optics (AO) systems in the context of a real synergy between researchers and engineers from different laboratories and institutes. The idea is to offer to AO community a fully operational

Further author information: (Send correspondence to A. Z.): E-mail: ziad@unice.fr, Telephone: (33) (0)4 92076338 
on-sky test platform. The ultimate goal being the optimization of the scientific returns of the AO assisted instrumentations.

The CATS station is also a support for our training activities as part of our Masters MAUCA and OPTICS, through the organization of on-sky practical works. We are also considering the organization of international summer schools and workshops on the techniques of Atmospheric Optics and Site-Testing in Astronomy around the CATS station.

\section{THE CALERN ATMOSPHERIC TURBULENCE STATION}

The CATS station is composed with a set of two autonomous and complementary instruments. The first one is the Generalized Differential Image Motion Monitor (GDIMM), 1 aiming at monitoring all the integrated parameters of the optical turbulence (seeing, isoplanatic angle, coherence time and outer scale). In order to avoid the turbulent surface layer, the GDIMM is installed on a 4 meters high concrete pilar (left of Fig 1). The instrument is protected by a $7 \mathrm{ft}$ allsky dome attached to a metallic tower. This tower also supports all the peripheral components of the GDIMM instruments (power management, electronics and computer). To minimize vibrations, the pilar foundation is isolated from the tower foundation. A particular attention was given to the stability of the ground below the instrument as the plateau is characterized by several karstic cavity (sinkholes and dolines). An electric tomography of the ground at the desired position of the station was done to locate possible defects in the underground.

The second instrument is the "Profiler of Moon Limb", 2 is devoted to the extraction of the $C_{n}^{2}(h)$ profile with high vertical resolution using the Moon or Solar limb fluctuations. This instrument is also protected by a $12 \mathrm{ft}$ allsky dome attached to a concrete platform (right of Fig 1). Finally, to make the CATS station works in an autonomous manner, an allsky camera to monitor the presence of clouds in the sky and a weather station are connected to a set of small computers (Banana $\mathrm{Pi}$ ) where devoted software runs to give real time information to both instruments for observation management.

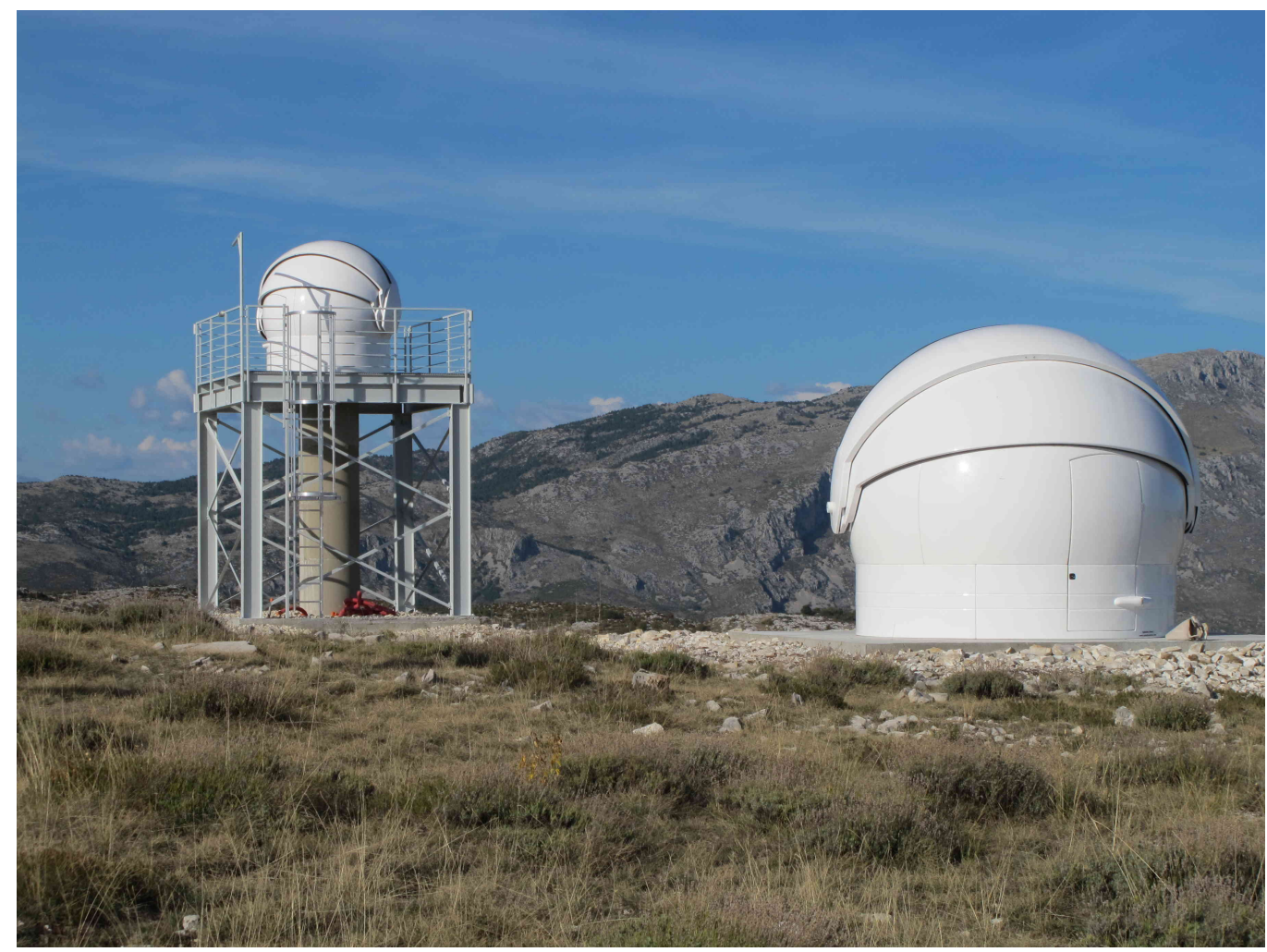

Figure 1. The CATS station installed near the MeO telescope at Calern Observatory. 


\section{GENERALIZED DIFFERENTIAL IMAGE MOTION MONITOR (GDIMM)}

\subsection{GDIMM theoretical background}

The seeing is one of the most important parameters describing wavefronts perturbed by atmospheric turbulence. The seeing is related to the resolution of the images and corresponds to the angular size of the FWHM of long-exposure images observed through the atmospheric turbulence. Seeing monitors are operated in major observatories such as ESO Paranal and the most known are DIMM ${ }^{3}$ and GSM ${ }^{4}$ The DIMM is a seeing monitor which is very popular because of its simplicity. It is based on a small telescope with an entrance pupil made of 2 small subapertures, observing a bright single star with a short exposure (typically a few miliseconds). A tilt is given to the light propagating through one of the two apertures to produce twin images which move according to the turbulence strength. Angular distances $\alpha$ and $\beta$ (called Angle-of-Arrival) of the centroid of each star image are computed in both directions ( $\alpha$ is parallel to the basis of the sub-apertures). Longitudinal and transversal differential variances ( $\sigma_{l}^{2}$ and $\sigma_{t}^{2}$ respectively) of $\alpha$ and $\beta$ are estimated on a sequence of $N$ instantaneous snapshots ( $N$ being typically several hundreds). From these differential variances, the seeing $\epsilon$ (in radian) is deduced using the following formulae:

$$
\epsilon_{l \mid t}=0.98(\cos z)^{-0.6}\left(\frac{D}{\lambda}\right)^{0.2}\left(\frac{\sigma_{l \mid t}^{2}}{K_{l \mid t}}\right)^{0.6}
$$

where,

$$
\begin{aligned}
& K_{l}=0.364\left(1-0.532 b^{-1 / 3}-0.024 b^{-7 / 3}\right) \\
& K_{t}=0.364\left(1-0.798 b^{-1 / 3}+0.018 b^{-7 / 3}\right)
\end{aligned}
$$

$B$ is the distance between the sub-apertures, $D$ their diameter, $b=B / D, z$ is the zenithal distance and $\lambda$ the wavelength, traditionally set to $500 \mathrm{~nm}$ as a standard. Two estimations of the seeing are obtained for a given sequence, they are supposed to be the almost identical (isotropic hypothesis) if the exposure time is enough short in regards to the turbulence coherence time.

The Fried parameter ${ }^{\sqrt{5}} r_{0}$ indicates the wavefront's coherence length. It is defined as the diameter of a circular area over which the wavefront aberration rms due to optical turbulence is equal to 1 radian. The Fried parameter is related to the seeing via the following formulae:

$$
\epsilon_{l \mid t}=0.98 \frac{\lambda}{r_{0, l \mid t}}
$$

the Fried parameter is a length giving in $m$ and it is a function of wavelength $\lambda$ varying as $\lambda^{6 / 5}$

Another important parameter characterizing the wavefronts perturbed by the turbulence, is the isoplanatic angle $\theta_{0}$. This latter can be estimated from the scintillation of a single star observed through a pupil of diameter $10 \mathrm{~cm}$ and a central obstruction of $4 \mathrm{~cm}$. The principle of this $\theta_{0}$ estimation method is based on the similarity of the theoretical expressions of $\theta_{0}$ and the scintillation index $s \underline{46} \theta_{0}$ is obtained in arcsec for a wavelength $\lambda=500 \mathrm{~nm}$ by the following formula

$$
\theta_{0}^{-5 / 3}=A(\cos z)^{-8 / 3} s
$$

where $A=14.87^{4}$ and $z$ is the zenithal angle of the observed star.

The most difficult parameter to measure is the wavefront coherence outer scale $\mathcal{L}_{0}$. In the case of the GDIMM instrument, we propose to use variances of Angle-of-Arrival (AA) fluctuations through different size sub-apertures 
by means of the von Karman model. The AA variance of the image of a star observed through a small aperture of diameter $D$ is given, in square radians by the following equation ${ }^{7}$

$$
\sigma_{D}^{2}=0.17 \lambda^{2} r_{0}^{-5 / 3}\left[D^{-1 / 3}-1.525 \mathcal{L}_{0}^{-1 / 3}\right]
$$

For isotropic turbulence, the variances in $x$ and $y$ directions are identical. The pupil of GDIMM has 3 apertures, two of diameter $D_{1}=6 \mathrm{~cm}$, one of diameter $D_{3}=10 \mathrm{~cm}$. The normalized variance difference represented by the following ratio

$$
\Re=\frac{\sigma_{D_{1}}^{2}-\sigma_{D_{3}}^{2}}{\sigma_{D_{1}}^{2}}=\frac{D_{1}^{-1 / 3}-D_{3}^{-1 / 3}}{D_{1}^{-1 / 3}-1.525 \mathcal{L}_{0}^{-1 / 3}}
$$

is used in the GDIMM to provide estimations of the outer scale $\mathcal{L}_{0}$. Since the GDIMM has two $6 \mathrm{~cm}$ subapertures, one can deduce two estimations of $\mathcal{L}_{0}$.

In the other hand, the coherence time $\tau_{0}$ relevant for adaptive optics and interferometry, as defined by Roddie ${ }^{8}$ is given by

$$
\tau_{0}=0.31 \frac{r_{0}}{\bar{v}}
$$

where $\bar{v}$, the effective wind speed, is a weighted average of the wind speed on the whole atmosphere ${ }^{8}$ We had shown ${ }^{9}$ that it is possible to derive the effective wind speed from the the temporal structure functions $D_{\alpha \mid \beta}(\tau)$ of the AA fluctuations, defined as

$$
D_{\alpha \mid \beta}(\tau)=\left\langle[\alpha|\beta(t)-\alpha| \beta(t+\tau)]^{2}\right\rangle
$$

where $\alpha$ (resp. $\beta$ ) stands for the AA fluctuations in the $x$ (resp. $y$ ) direction (parallel to the right ascension (resp. declination)). The brackets \langle\rangle stand for temporal average. The AA is computed as the angular photocenter of the images produced by each sub-aperture. This function is zero for $\tau=0$ and saturates to a value $D_{\text {sat }}$ for $\tau \longrightarrow \infty$. We define its characteristic time $\tau_{\alpha \mid \beta}$ as the value of $\tau$ for which

$$
D_{\alpha \mid \beta}\left(\tau_{\alpha \mid \beta}\right)=\frac{D_{\mathrm{sat}}}{e}
$$

$\tau_{\alpha \mid \beta}$ is indeed the AA coherence time. The effective wind speed as well as its direction $\gamma$ are derived from $\tau_{\alpha}$ and $\tau_{\beta}$ using eqs. 10 and 11 of $\left[9\right.$, taking $k^{\prime}=e$. This effective wind is then, combined with the Fried parameter $r_{0}$ in eq.7 to provide estimations of the coherence time $\tau_{0}$.

\subsection{GDIMM instrument}

The J.L Lagrange Laboratory has developed the GDIMM monitor, a compact instrument aiming at replacing the aging Generalized Seeing Monitor (GSM) 490 GDIMM is very similar to a DIMM, $\frac{10}{10}$ with 3 sub-apertures instead of 2. It has been developed using the experience on the GSM instrument ${ }^{4}$ and of the qualification of the Dome C site in Antartica. 11

The GDIMM instrument is composed by a Schmidt-Cassegrain Celestron 11 telescope (diameter $280 \mathrm{~mm}$ ) equipped with an entrance mask with 3 sub-pupils as shown in Fig. 2, It is is derived from the classical 2apertures DIMM mask, with a supplementary sub-pupil used for estimating the isoplanatic angle and the outer scale. The two main sub-pupils are circular with a diameter of $6 \mathrm{~cm}$, they are both equipped with a glass prism with a deviation angle of about 30 arcsec. The prims are oriented to give opposite tilts to the incident light. The mak is oriented so that the aperture separation is parallel to the declination axis. The third sub-aperture is also circular, with a $10 \mathrm{~cm}$ diameter and a $4 \mathrm{~cm}$ central obstruction. This aperture is left open and the corresponding image forms on the optical axis. The telescope is placed on an Astro-Physics 900 equatorial mount remotely controlled by a computer via a RS-232 link. In the focal plane, a Prosilica EC650 camera, captures the three 
star image at a maximum frame rate is $90 \mathrm{fps}$ at full resolution (windowing and binning options are available to increase the frame rate if necessary). A Barlow lens increases the focal length to 7.1 meters to allow a slight oversampling of the Airy discs (5 pixels at a wavelength of $500 \mathrm{~nm}$ in the Airy disc of the $10 \mathrm{~cm}$ diameter sub-pupil). This "science" camera is connected to the computer via an Ethernet cable. Finally, a second camera (USB webcam Logitech 9000) is placed at the focus of a Celestron Travelscope 90, used as a finder with a wide field of 4 degrees. This camera is sensitive enough to detect all the bright stars (Mag $<2$ ) used for the GDIMM monitoring of the turbulence.

The image given by the science camera are treated by homemade acquisition software written in $\mathrm{C}++/ \mathrm{QT}$ which takes benefit of years of developments of automated instruments for the Antarctic. It can point the mount to the desired star, detects and centers automatically the target on the finder and on the science camera. Observations can be made manually or by acquisition sequences. Seeing, isoplanatic angle, coherence time and outer scale are computed in real time. Various tests are performed to stop the observations when the star is lost (clouds) or if its zenithal distance becomes too large. Data are written in text-based csv files but it is also possible to record the images in FITS cubes. Each measurement is finally sent to a website available for the users of the observatory (see Fig 6 ).
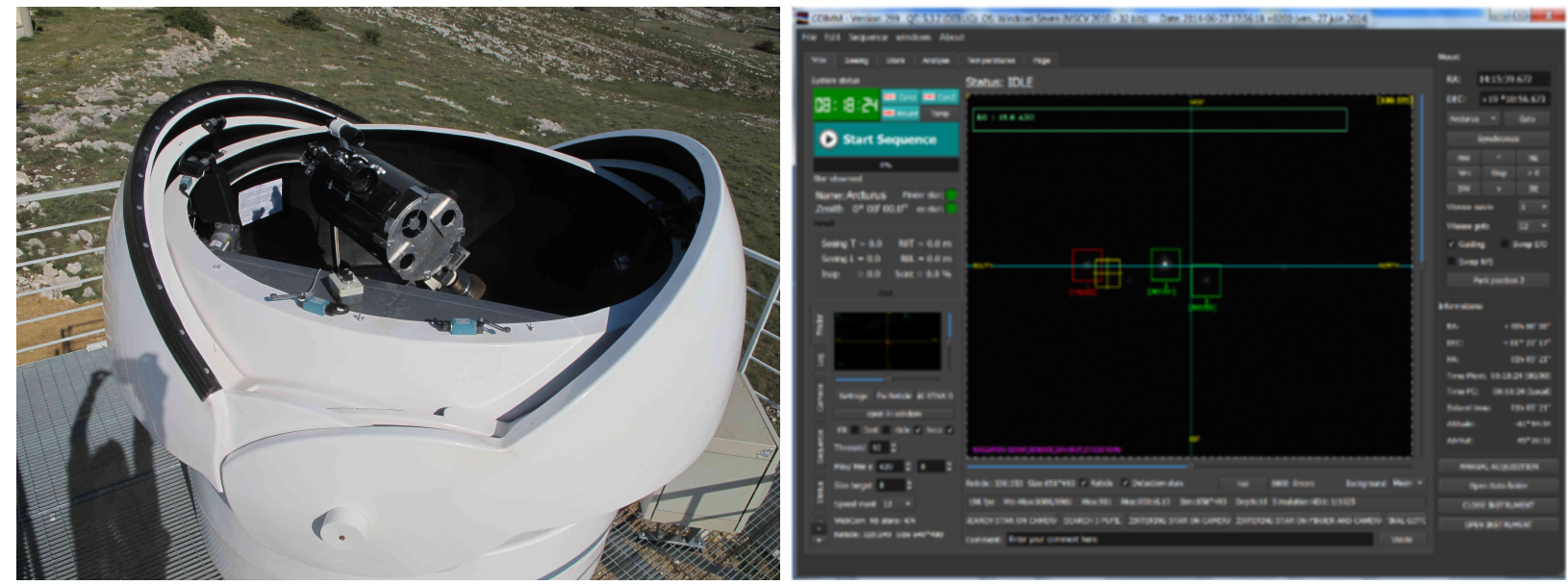

Figure 2. Left: The GDIMM instrument inside its $7 \mathrm{ft}$ allsky dome. Right: The GDIMM acquisition software.

\section{PROFILER OF MOON LIMB (PML)}

The PML instrument is dediceted to the extraction of the $C_{n}^{2}$ profile with high vertical resolution from lunar (or solar) limb fluctuations. The PML instrument is based on a differential method by observation of the lunar (or solar) limb through two sub-apertures (Fig. 3). The Moon or Solar limb acts as a contiunuum of double stars with all possible angular separations required between two points to scan the atmosphere with a very fine resolution. The PML instrument uses the differential method of the DIMM through two subapertures of $6 \mathrm{~cm}$ diameter separated by a baseline of $26.7 \mathrm{~cm}$. The principle of the PML instrument is based on the measurement of the angular correlation of the fluctuation differences in the wavefront angle-of-arrival (AA) deduced from the motion of the Moon's (or sun) limb image. The AA fluctuations are measured perpendicularly to the lunar (or solar) limb leading to transverse correlations for different angular separations along the Moon (or sun). The angular correlation along the lunar (or solar) limb of the differential distance between the two lunar (or solar) edges leads to the $C_{n}^{2}(h)$ profile. Other parameters of turbulence are also accessible from this instrument such as the profile of outer scale, the seeing, and the isoplanatic and isopistonic angles.

\subsection{PML theoretical background}

The observation of the lunar (or solar) limb through two sub-apertures of diameter $D=6 \mathrm{~cm}$ separated by a baseline $B=26.7 \mathrm{~cm}$ presents two configurations when looking the edge in parallel or perpendicular to the baseline. We use the first configuration to extract the $C_{N}^{2}$ vertical distribution. 
The transverse covariance of the difference of the AA fluctuations (motion of the Moon or Sun limb) $\alpha$ between the two images of the lunar (or solar) limb (Fig. 3) corresponds to,

$$
C_{\Delta \alpha}(\theta)=\left\langle\left[\alpha\left(r, \theta_{0}\right)-\alpha\left(r+B, \theta_{0}\right)\right]\left[\alpha\left(r, \theta+\theta_{0}\right)-\alpha\left(r+B, \theta+\theta_{0}\right)\right]\right\rangle
$$

where $\alpha\left(r, \theta_{0}\right)$ and $\alpha\left(r, \theta+\theta_{0}\right)$ represent the fluctuations of the lunar (or solar) limb image observed through the first subaperture of the PML and measured at the angular positions $\theta_{0}$ and $\theta+\theta_{0}$, respectively. While, $\alpha\left(r+B, \theta_{0}\right)$ and $\alpha\left(r+B, \theta+\theta_{0}\right)$ are the measured fluctuations corresponding to the second subaperture. The arbitrary angular position $\theta_{0}$ is considered equal to zero.

After development, this expression is a function of the spatial covariance which for the whole atmosphere is given by,

$$
C_{\Delta \alpha}(\theta)=\int d h C_{N}^{2}(h) K_{\alpha}(B, h, \theta)
$$

where

$$
K_{\alpha}(B, h, \theta)=2 C_{\alpha}(\theta h)-C_{\alpha}(B-\theta h)-C_{\alpha}(B+\theta h)
$$

In this equation $C_{\alpha}$ is the normalized spatial covariance which in the case of the von Kàrmàn model for a baseline $\varrho$, a sub-aperture diameter $\mathrm{D}$ (here $6 \mathrm{~cm}$ ), and a single layer at altitude $h$ is given by ${ }^{12}$ as

$$
C_{\alpha}(\varrho)=1.19 \sec (z) \int d f f^{3}\left(f^{2}+\frac{1}{\mathcal{L}_{0}(h)^{2}}\right)^{-11 / 6}\left[J_{0}(2 \pi f \varrho)+J_{2}(2 \pi f \varrho)\right]\left[2 \frac{J_{1}(\pi D f)}{\pi D f}\right]^{2}
$$

where $f$ is the modulus of the spatial frequency, $z$ is the zenithal distance and $\mathcal{L}_{0}(h)$ is the outer scale profile.

Eq 11 represents for a single layer a spatial covariance triplet similar to the Scidar one 13 The location of the lateral peak defines the altitude of the layer so that its energy is given by the height of it. For the whole atmosphere we have the superposition of different triplets corresponding to different turbulent layers.

Eq. 12 is non-linear but analytical solution of the integral expression has been made by Conan et al 14 through the use of Mellin transform. Resulting covariances are simplified into series or finite solutions. Approximations however are different depending on the baseline length. We used these approximations and the assumption of a discrete turbulent profile to transform eq] 10 into

$$
C_{\Delta \alpha}(\theta)=\sum \Delta h_{i} C_{N}^{2}(h) \widehat{K}_{\alpha}\left(B, h_{i}, \theta\right)
$$

where $\widehat{K}_{\alpha}\left(B, h_{i}, \theta\right)$ is the modified spatial covariance triplet and $\Delta h_{i}$ is the thickness of the layer i. Eq. 13 is equivalent to a matrix form: $Y=M . X$ where $\mathrm{X}$ and $\mathrm{Y}$ are both vectors corresponding respectively to the sampled $C_{N}^{2}\left(h_{i}\right)$ and the covariance difference $C_{\Delta \alpha}(\theta)$, the matrix M contain the modified spatial covariance triplet weighted with $\Delta h_{i}: M=\widehat{K}_{\alpha}\left(B, h_{i}, \theta_{j}\right) . \Delta h_{i}$.

The $C_{N}^{2}(h)$ profiles are retrieved by solving an inverse problem via minimization of a maximum likelihood criterion under positivity constraint using an iterative gradient method ${ }^{15}$ Another approach using a regularization method has been also studied. In addition, we also used a simulated annealing algorithm for the minimization process leading to the reconstruction of the turbulence profile $C_{N}^{2}(h)$ 16 17 PML instrument errors are mainly related to the detection of the Moon limb position and are mostly due to photon noise. 


\subsection{PML instrument}

The PML instrument consists of a 16-inch telescope (Meade M16) installed on an Astrophysics AP3600 mount. The pupil mask composed of two sub-apertures of diameter $D=6 \mathrm{~cm}$ separated by a base-line $B=26.7 \mathrm{~cm}$, is placed at the entrance pupil of the telescope. An optical system is installed at the ouput of the telescope. It consists of a collimated beam by using a first lens L1 placed at its focal length from the telescope focus (Figs. 3 and 4. Then, two parallel beams are formed at the output of L1 corresponding to each sub-aperture. A Dove prism is inserted on one of the two beams to reverse one of two images of the lunar edge in order to avoid an overlapping of the two image of Moon (Fig. 3). A second lens L2 is used to form the two images of the Moon limb on the CCD camera. Each optical element is placed on a Micro-control plate allowing fine adjustments. To compensate for variations in the telescope's focus because of the temperature variations, we installed the CCD camera on an automatic micro-control plate controlled by the acquisition software (Fig. 4). Images at the focal plane are recorded using a PixelFly CCD camera with $640 \times 480$ pixel matrix. In order to freeze atmospheric effects on the motion of the Moon's limb image and to have enough flux, the exposure time is set to $5 \mathrm{~ms}$.

The acquisition software, similar to GDIMM's one, extracts the AA fluctuations of both lunar limb and save them as .csv files. The theoretical background for the computation of the $C_{n}^{2}(h)$ profile from the AA fluctuations is given in Ziad et al ${ }^{2]} \mathrm{A}$ real time computation of this profile is currently under development. A computational server will be in charge of the computation of the $C_{n}^{2}(h)$ profile. We expect to be able to generate a high resolution vertical profile of $C_{n}^{2}$ every minute in the next few months. In addition, the PML instrument will be equipped soon with automatic panels to cover the two subapertures with Solar filters for a fast and automatic switch from night/Moon observation to day/Solar observation. We will have then, a unique tool to study the turbulence conditions at the daytime to nightime transition.
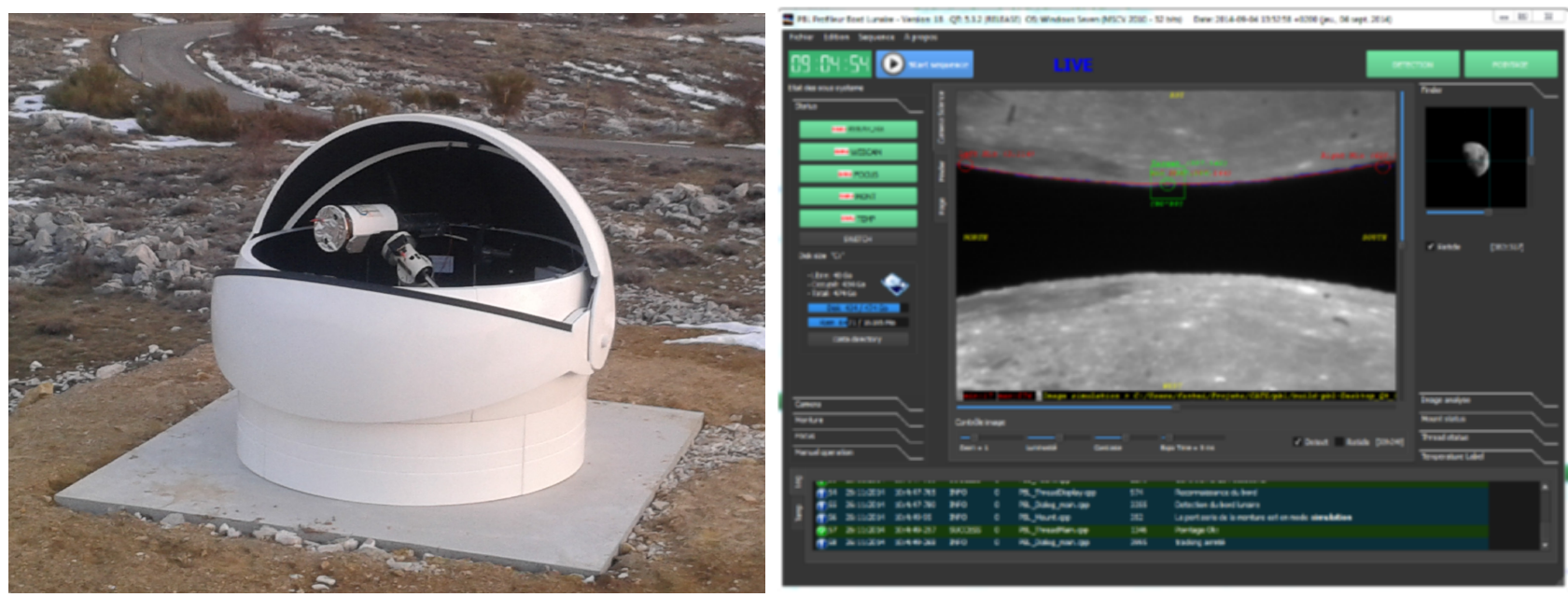

Figure 3. Left: the Profiler of Moon Limb instrument inside its 12ft allsky dome. Right: the graphical user interface of the PML instrument.

\section{CATS: AN AUTONOMOUS STATION}

The CATS station has been designed to be autonomous without human intervention. Indeed, CATS is equipped with an allsky camera to detect the presence of clouds in the sky and a weather station for monitoring, temperature, humidity, dew point and wind velocity. All these sensors information is treated by a software devoted to observation management.

The all sky camera dedicated to the clouds cover estimation is is an Oculus camera that provides high quality sky images. It has highly sensitive Sony ICX205AL CCD camera with low noise electronics and $150^{\circ}$ fisheye lens. The camera is connected to a computer where a dedicated homemade software analyzes the nigh image to compute the fraction of sky occupied by clouds. It operates all night and updates an image on the data website every minute (Fig. 6). 

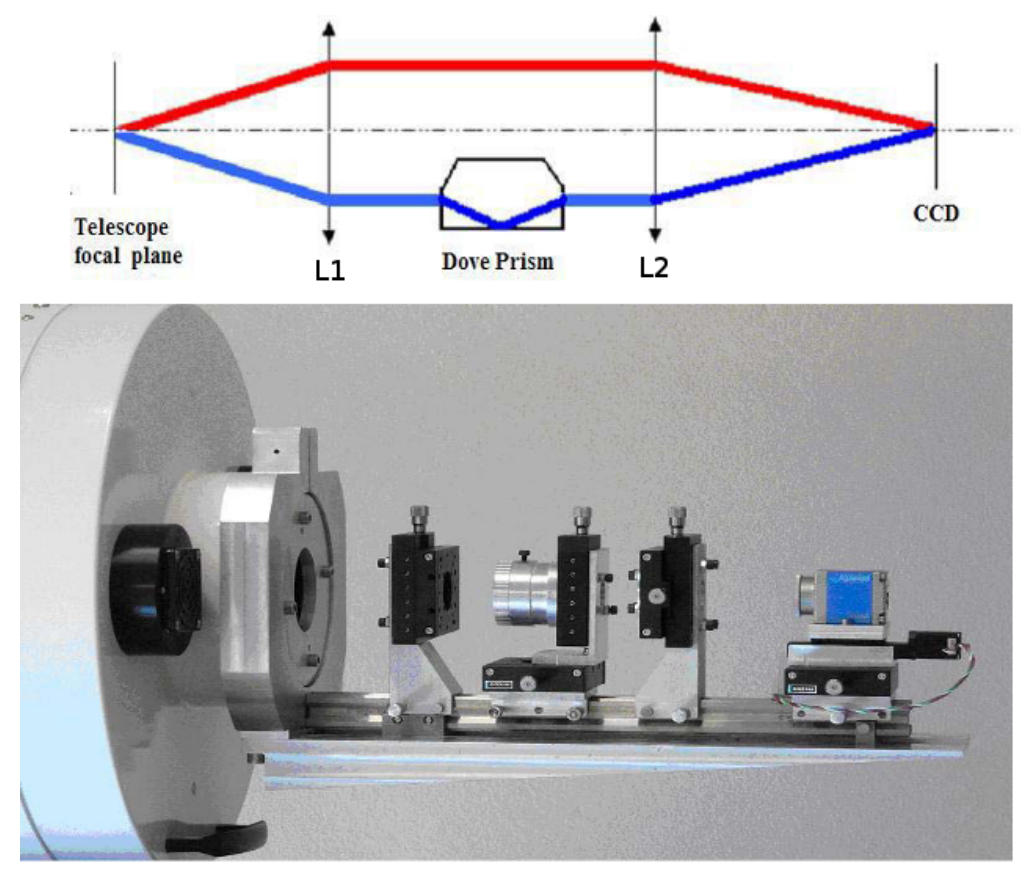

Figure 4. Optical device of the PML instrument.

In addition, the CATS station is also equipped with a weather station which is commercial Vantage pro 2 connected to a Banana-Pi computer. It provides all the necessary quantities (temperature, humidity, pressure, dew point, wind speed and rain gauge) for a constant monitoring.

A small software called "cats weather" (Fig. 5 ) has been written in python to gather all the data from the allsky camera software (fraction of sky covered by clouds) and from the weather station. From this data, the software determines if the conditions are good enough to let the two instruments open their domes and start their observation. If the sky becomes too cloudy or if outside temperature becomes too close of the dewpoint temperature, the software forces to both domes to close in order to protect instruments from rain or fog. If the wind speed is above $30 \mathrm{~km} / \mathrm{h}$, observations are stopped and domes are closed, as both instruments are shaken and no data can be acquired.

\section{CATS RESULTS}

\subsection{Why Calern Observatory?}

The Calern Observatory was inaugurated in 1974 under the name "Centre d'Étude et de Recherches en Géodynamique et Astronomie" (CERGA). It was designed from the beginning as a laboratory intended for the development and testing of new instruments. The essential qualities of this $20 \mathrm{~km}^{2}$ semidesertic calcareous plateau are a high number of clear nights, an absence of mists and dusts, a not too dry atmosphere, and a circulation of winds in horizontal layers due to the topography and to the presence of the marine wind which stabilizes the atmosphere.

Since 1988, the CERGA has merged with the Observatoire de Nice (French Riviera) to give birth to the Observatoire de la Côte d'Azur (OCA), the plateau of Calern is the site of observation of the OCA. From the 70's to the 90's, the Calern Observatory was the place of birth of many precursory instruments in their discipline, in particular in laser telemetry and interferometry with two telescopes (I2T) and its successor GI2T. The activity of the Schmidt telescope with the large field imaging of small objects was also very intense up to the early 2000s. Nowadays, the scientific activity of the plateau continues with projects which exploit the acquired experience, and the particular links between astronomy and geophysics: Satellite Laser Ranging, Lunar Laser Ranging, Time Transfert by Laser Link with the T2L2 space mission, Spatial Geodesy, Measure of the Solar diameter with PICARD-SOL, follow on project of fast transitory objects (TAROT), asteroids photometry and polarimetry (CAPS), double star characterization (PISCO), giant planet sismology (DSI), follow-up of GAIA 


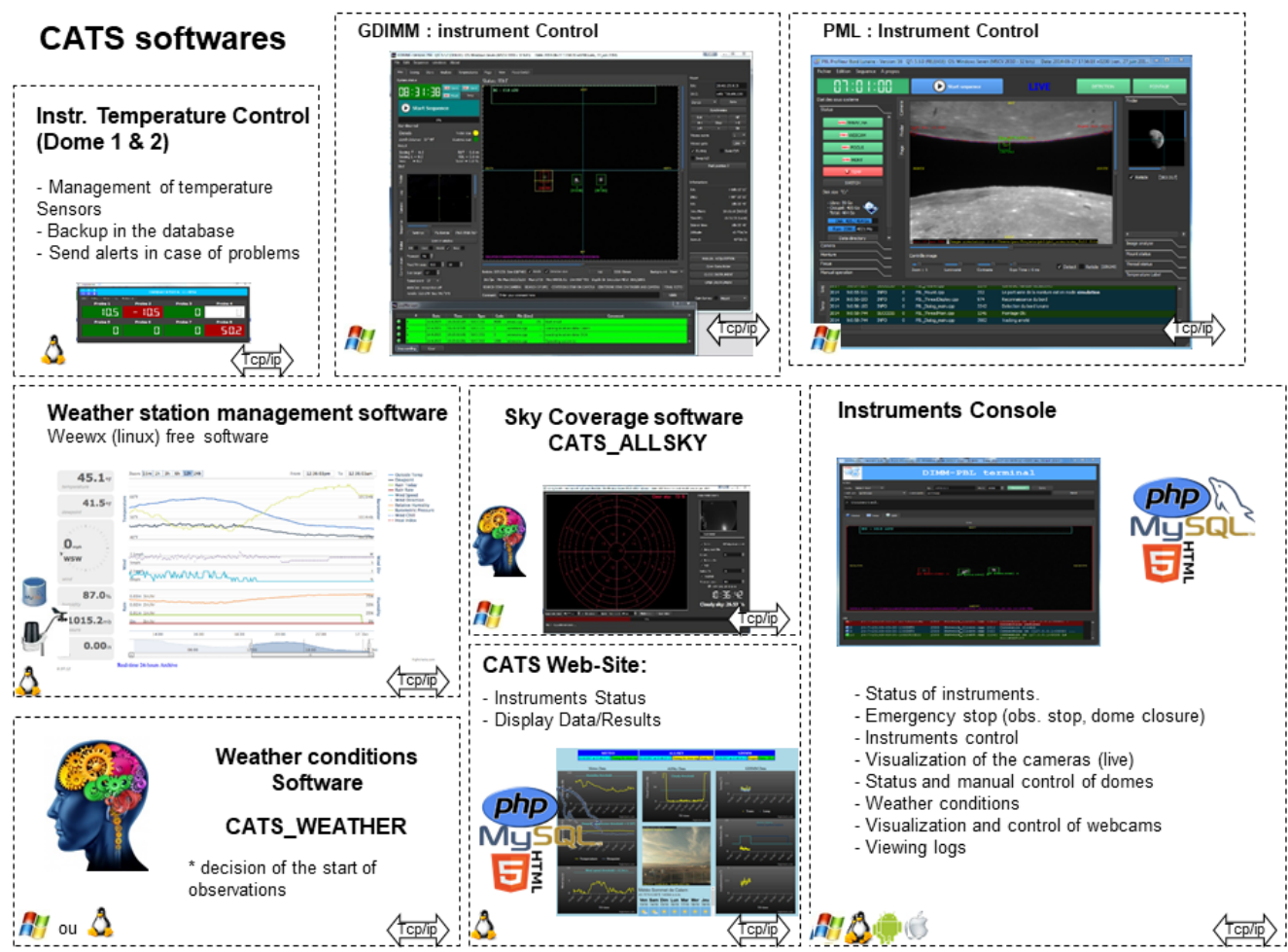

Figure 5. The structure of softs dedicated to CATS observation management.

space telescope alerts. Several projects with industrial partners like Airbus Defence and Space (Geotracker), Thales Alenia Space, or ONERA on adaptive optics are carried on. Recently, the Calern plateau host teaching and training activities for master students through an infrastructure devoted to the immersion of students in the experimental environment in astrophysics and geophysics, the Center for Pedagogy in Planet and Universe sciences (C2PU). All these activities needs a permanent and reliable monitoring of the turbulence conditions above the plateau of Calern, now done by the CATS instruments.

\subsection{GDIMM Data}

Observations with GDIMM are composed of continuous sequences of less than 1 minute of time, each sequence giving one set of turbulence parameters: seeing $\epsilon$ (two values are calculated, longitudinal $\epsilon_{t}$ and transverse $\epsilon_{l}$ ), isoplanatic angle $\theta_{0}$, outer scale $\mathcal{L}_{0}$ and coherence time $\tau_{0}$. The acquisition software computes in real time all these atmospheric turbulence parameters which are sent to CATS website and displayed on the screen as shown in Fig. 6. The CATS station is operational since mid-October 2015. Fig. 7 shows a summary of the results obtained during 1.5 year with CATS. The seeing is about $1^{\prime \prime}$ which represents the median value for this site that may show nights with low seeing values but in lesser quantity than an excellent site.

\subsection{PML results}

Fig. 8 shows an example of the turbulence profile evolution throughout the night on 2011 August 11 from PML measurements at the SAAO Sutherland Observatory in South Africa. The figure shows the full PML profile with 33 layers. The resolution obtained by the PML is $\Delta h=100 \mathrm{~m}$ for the ground layer $(h \leq 1 \mathrm{~km}), \Delta h=500 \mathrm{~m}$ for the low free atmosphere $(1 \mathrm{~km}<h<5 \mathrm{~km}), \Delta h=1000 \mathrm{~m}$ for the mid-free atmosphere $(5 \mathrm{~km}<h<15 \mathrm{~km})$, and $\Delta h=2000 \mathrm{~m}$ for the high free atmosphere $(h>15 \mathrm{~km})$. The highest altitude $h_{\text {max }}$ measured with the PML is more than $50 \mathrm{~km}$. However, we limited $h_{\max }$ to $25 \mathrm{~km}$ since the turbulence is very low beyond this altitude. On the other hand, because of a limited field of view the PML instrument has a minimum altitude detectable which is around $100 \mathrm{~m}$. The contribution of the lowest layer $0-100 \mathrm{~m}$ is obtained by the difference between the profile 


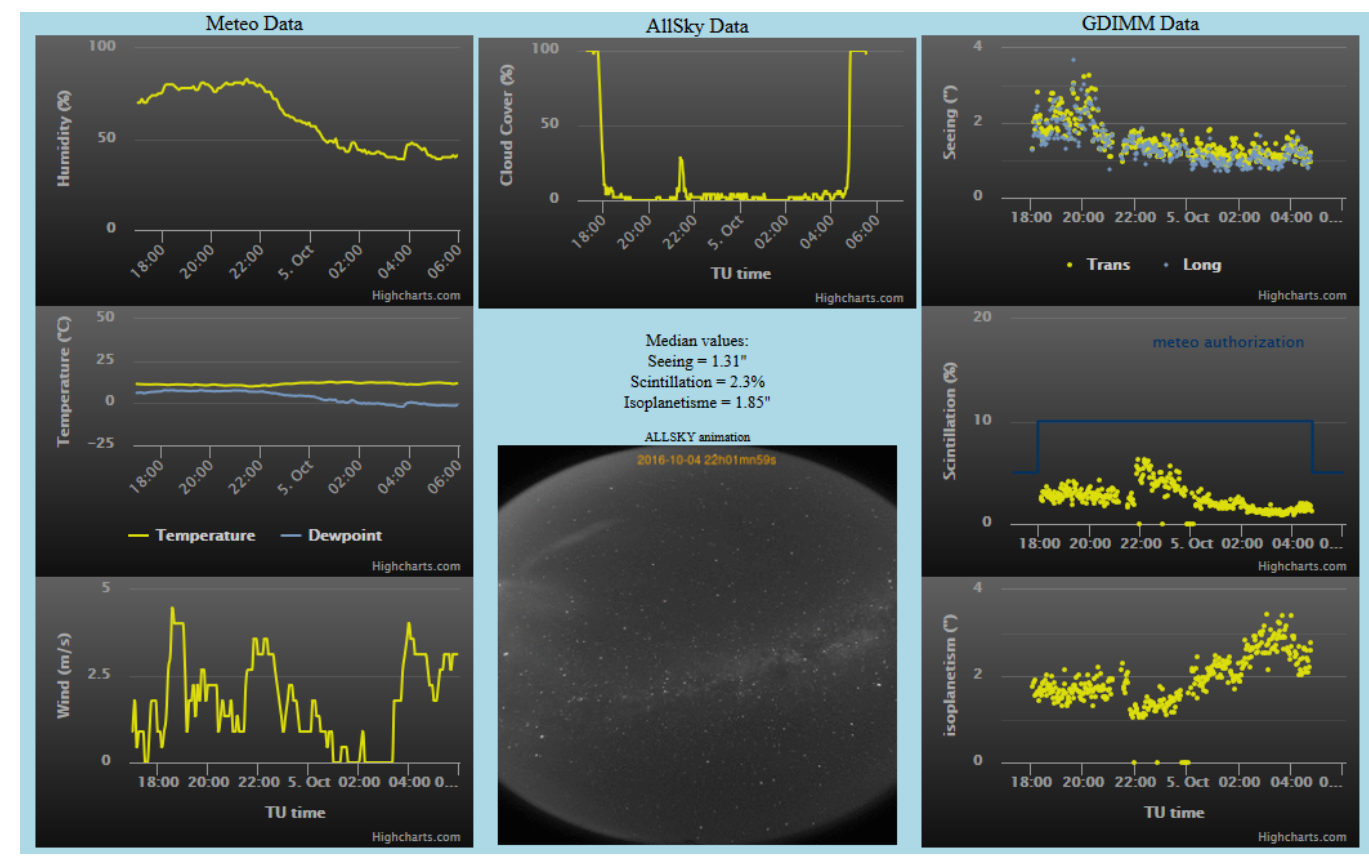

Figure 6. The GDIMM real time data website.
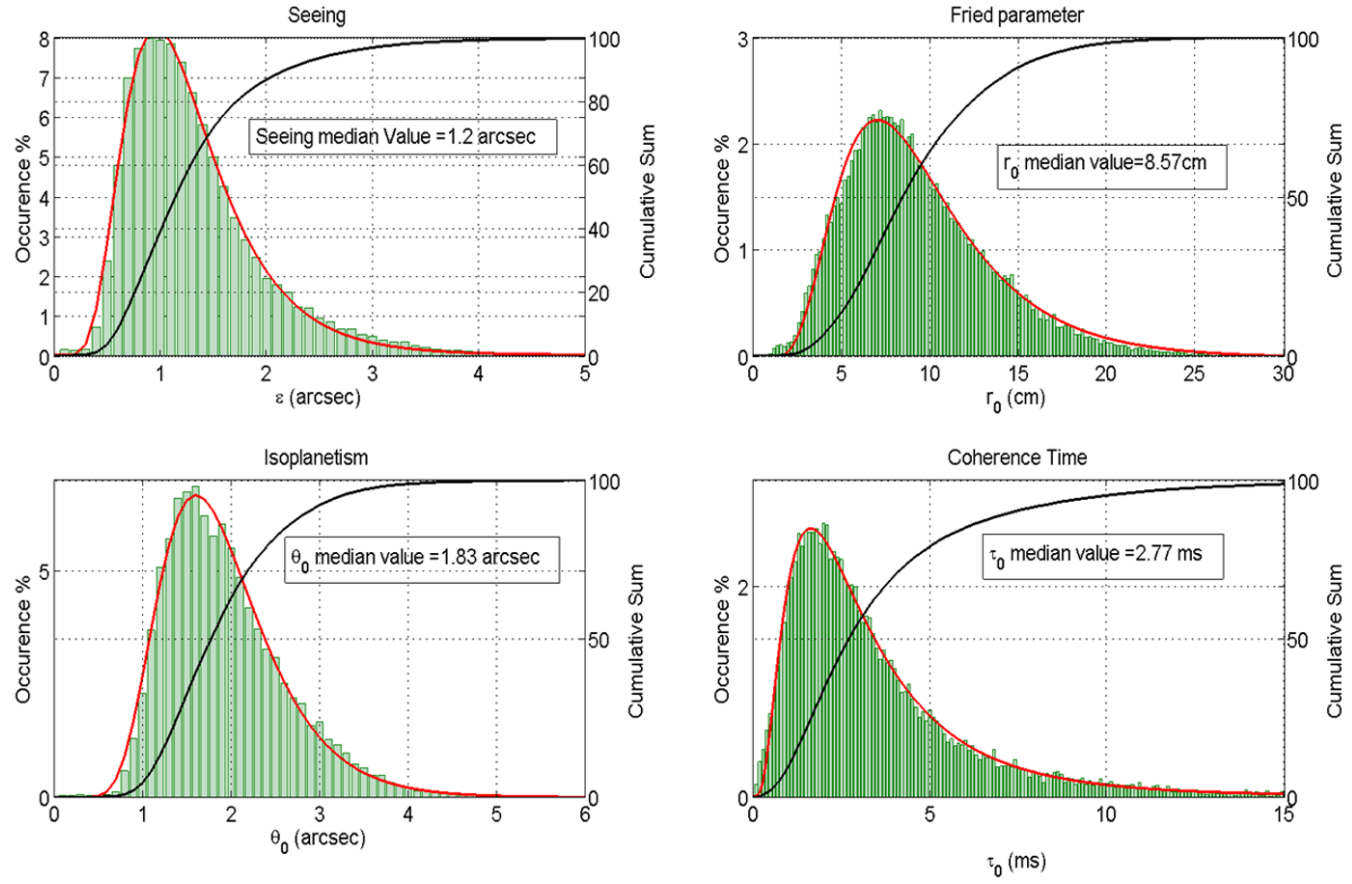

Figure 7. The GDIMM Statistics on data accumulated since October 2015 .

deduced from the inversion of the PML covariances and the total seeing from DIMM method (sect. 3) using PML data. For the total seeing obtained from PML, we have about 620 estimations (each point of the Moon limb leads to a DIMM measurement) and we keep only the median one. The PML instrument provides also other parameters of turbulence such as the profile of outer scale, the seeing, and the isoplanatic and isopistonic angles. 


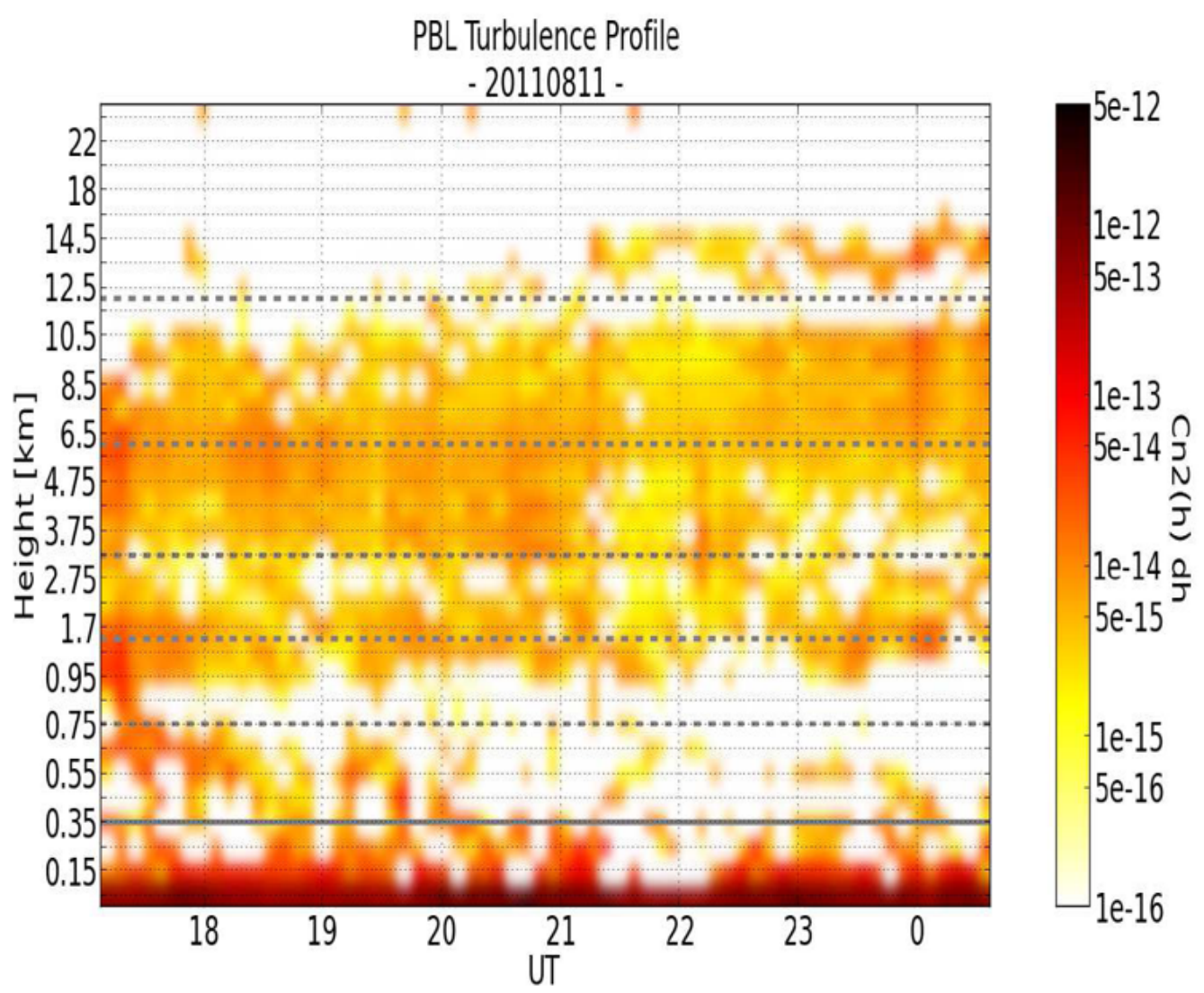

Figure 8. Turbulence profiles for the night of 2011 August 11 with the PML instrument at the SAAO Sutherland Observatory in South Africa.

\section{DISCUSSION}

The CATS station is installed and operational at the Calern Observatory since autumn 2015. CATS is a new generation station of atmospheric turbulence measurement which is equipped with a set of complementary instruments for monitoring atmospheric turbulence parameters. The CATS station is completely autonomous without human intervention for a fully monitoring of the turbulence within original techniques since the first meters above the ground to the borders of the atmosphere. CATS station provides a real-time turbulence conditions over the Calern Observatory. The CATS station is a support involved in many scientific and educational projects at the Calern observatory such as an ongoing study of turbulence impact on the laser links of the MeO Laser Ranging Station, development of Adaptive Optics projects. Indeed, different campaigns involving optical telecommunication from space to ground with the SOTA satellite and MéO laser ranging station has already

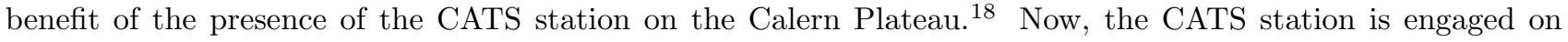
a campaign with the space instrument T2L2 and the MéO laser station to sudy the impact of turbulence on time transfert by laser link (T2L2). It is the very first time that a permanent instrumentation at the ground is installed next to a laser ranging station and associated with a space instrument to study the turbulence effects on laser link budget from ground to space ${ }^{19}$ Another campaign has been performed with the Optical PAyload for Lasercomm Science (OPALS) on International Space Station in spring 2016. The PML instrument will be very useful for understand the link budget of the lunar laser ranging activity at the MéO telescope. Several projects regarding adaptive optics systems are planned at the MéO telescope and C2PU telescope and they will benefit of the data given by the CATS station. A portable GDIMM instrument will be involved in the qualification of a site for a new geodesic observatory at Tahiti. Finally, the CATS station is used for on sky practical work for students for the OPTICS master and for the international Master in Astrophysics of Université Côte d'Azur (MAUCA). 


\section{ACKNOWLEDGMENTS}

This CATS project has been done under the financial support of CNES, Observatoire de la Côte d'Azur (OCA), Labex First TF, AS-GRAM, Federation Doblin, Université de Nice-Sophia Antipolis and Region Provence Alpes Côte d'Azur. We would like thanks all the technical and administrative staff of the Observatoire de la Côte d'Azur and our colleagues from the Astrogéo team from GéoAzur laboratory for their help and support all along the project namely Pierre Exertier, Etienne Samain, Dominique Albanèse, Mourad Aimar, Jean-Marie Torre, Emmanuel Tric, Thomas Lebourg and Sandrine Bertetic.

\section{REFERENCES}

[1] Aristidi, E., Fanteï-Caujolle, Y., Ziad, A., C., D., Chabé, J., and Roland, B., "A new generalized differential image motion monitor," Astronomical Telescopes+ Instrumentation, Proc. SPIE 9145, 91453G (2014).

[2] Ziad, A., Blary, F., Borgnino, J., Fantei-Caujolle, Y., Aristidi, E., Martin, F., Lanteri, H., Douet, R., Bondoux, E., and Merkarnia, D., "First results of the pml monitor of atmospheric turbulence profile with high vertical resolution," $A \mathscr{E} A$ 559, L6 (2013).

[3] Sarazin, M. and Roddier, F., "The eso differential image motion monitor," Astronomy and Astrophysics, vol. 227, no. 1, Jan. 1990, p. 294-300. (1990).

[4] Ziad, A., Conan, R., Tokovinin, A., Martin, F., and Borgnino, J. Applied Optics 39, 5415 (2000).

[5] FRIED, D. L., "Optical resolution through a randomly inhomogeneous medium for very long and very short exposures," J. Opt. Soc. Am. 56, 1372-1379 (Oct 1966).

[6] Loos, G. and Hogge, C. Applied Optics 18, 15 (1979).

[7] Ziad, A., Borgnino, J., Martin, F., and Agabi, A., "Experimental estimation of the spatial-coherence outer scale from a wavefront statistical analysis," AËA 282, 1021-1033 (feb 1994).

[8] Roddier, F., Gilli, J., and Lund, G. J. Opt. (Paris) 13, 263 (1982).

[9] Ziad, A., Borgnino, J., Ali, W. D., Berdja, A., Maire, J., and Martin, F., "Temporal characterization of atmospheric turbulence with the generalized seeing monitor instrument," Journal of Optics 14(4), 045705 (2012).

[10] Tokovinin, A., "From differential image motion to seeing," Publications of the Astronomical Society of the Pacific 114, 1156 (2002).

[11] Aristidi, E., Agabi, A., Fossat, E., Azouit, M., Martin, F., Sadibekova, T., Travouillon, T., Vernin, J., and Ziad, A., "Site testing in summer at dome c, antarctica," A\&A 444(2), 651-659 (2005).

[12] Avila, R., Ziad, A., Borgnino, J., Martin, F., Agabi, A., and Tokovinin, A., "Theoretical spatiotemporal analysis of angle of arrival induced by atmospheric turbulence as observed with the grating scale monitor experiment," J. Opt. Soc. Am. A 14, 3070-3082 (Nov 1997).

[13] Fuchs, A., Tallon, M., and Vernin, J. PASP 110, 86 (1998).

[14] Conan, R., Borgnino, J., Ziad, A., and Martin, F. J. Opt. Soc. Am. 17(10), 1807 (2000).

[15] Blary, F., Caractérisation et Modélisation de la Turbulence Optique dans un espace confiné, PhD thesis, Université de Nice Sophia Antipolis (2015).

[16] Maire, J., Ziad, A., Borgnino, J., and Martin, F. MNRAS 377, 232 (2007).

[17] Catala, L., Ziad, A., Fanteï-Caujolle, Y., Crawford, S. M., Buckley, D. A. H., Borgnino, J., Blary, F., Nickola, M., and Pickering, T. MNRAS 467, 3699 (2017).

[18] Vedrenne, N., Velluet, M.-T., Petit, C., Michau, V., Phung, D.-H., Maurice, N., Samain, E., Chabé, J., Ziad, A., Artaud, G., Issler, J.-L., Toyoshima, M., Akioka, M., Kolev, D. R., Munemasa, Y., Takenaka, H., and Iwakiri, N., "First results of wavefront sensing on SOTA," in [2015 IEEE International Conference on Space Optical Systems and Applications (ICSOS2015)], (Oct. 2015).

[19] Sinclair, L. C., Giorgetta, F. R., Swann, W. C., Baumann, E., Coddington, I., and Newbury, N. R., "Optical phase noise from atmospheric fluctuations and its impact on optical time-frequency transfer," Phys. Rev. A 89, 023805 (Feb 2014). 